\title{
Mice with Asthma Are More Resistant to Influenza Virus Infection and NK Cells Activated by the Induction of Asthma Have Potentially Protective Effects
}

\author{
Hiroki Ishikawa • Hiraku Sasaki • Toshie Fukui • \\ Koji Fujita • Etsuko Kutsukake • Tetsuya Matsumoto
}

Received: 16 September 2011 / Accepted: 14 November 2011 /Published online: 2 December 2011

(C) The Author(s) 2011. This article is published with open access at Springerlink.com

\begin{abstract}
Purpose This study was conducted in order to investigate whether the virulence of the influenza virus infection is affected by asthma in mice.

Methods Mice with asthma or control mice were infected with influenza virus. The survival rate, body weight, virus titer, cytokine profile, and cell infiltration in bronchoalveolar lavage fluid (BALF) were measured. The NK cell cytotoxicity was determined by a co-culture system with YAC-1 cells, and the effects of NK cells were observed by depletion of NK cells using anti-asialoGM1 serum. The virus-specific $\mathrm{CD} 8^{+} \mathrm{T}$ cell killing assay was also performed.

Results When asthmatic or control mice were infected with non- and sub-lethal doses of influenza virus, the asthmatic mice were more resistant to the virus than control mice with regard to the survival rate, the remission of body weight loss, and the virus burden. Anti-viral cytokines and the NK cell number were increased in the BALF of asthmatic mice before the infection. The NK cell cytotoxicity in the asthmatic mice was significantly enhanced compared to that in control mice, and the depletion of NK cells in asthmatic mice was abrogated both the improved
\end{abstract}

H. Ishikawa $(\bowtie) \cdot$ T. Fukui $\cdot$ E. Kutsukake · T. Matsumoto

Department of Microbiology, Tokyo Medical University,

6-1-1 Shinjuku, Shinjuku-ku,

Tokyo 160-8402, Japan

e-mail: hiroki01@tokyo-med.ac.jp

H. Sasaki

Animal Research Center, Tokyo Medical University,

Shinjuku-ku,

Tokyo 160-8402, Japan

K. Fujita

Department of Molecular Pathology, Tokyo Medical University,

Shinjuku-ku,

Tokyo 160-8402, Japan survival rate and the recovery of the body weight loss. The antigen-specific $\mathrm{CD}^{+} \mathrm{T}$ cell killing activity in asthmatic mice was also significantly increased following the infection compared to that in control mice.

Conclusion NK cell activated by the induction of asthma and the subsequently activated antigen-specific $\mathrm{CD}^{+} \mathrm{T}$ cells could promptly eliminate the viral-infected cells, thus leading to improvements in the morbidity and mortality of influenza virus infection.

Keywords Asthmatic mice - influenza virus · NK cell . antigen-specific $\mathrm{CD}^{+} \mathrm{T}$ cell

\section{Introduction}

At least one strain of influenza virus infects people worldwide every year. The virus infects the upper and lower respiratory tract resulting in various symptoms, such as sudden fever, cough, and headache. The pathogenesis of the influenza virus infection is influenced by the host's genetic, immune, physical, and viral factors [1-3]. Among these factors, an epidemiological study revealed that asthma is one of the physical risk factors for a complicated clinical condition resulting from influenza virus infection $[4,5]$. On the other hand, it has been reported that hospitalized patients with asthma are less likely to die due to influenza A virus infection [6]. The reason for these contradictory findings is unclear. Therefore, the aims of this study were to investigate whether the pathogenesis of influenza virus infection is affected by asthma in a mouse model and to reveal the immunological mechanisms underlying these effects in vivo.

The prevalence of patients with asthma has dramatically increased in the last few decades, and asthma now affects 
more than 300 million people worldwide, especially in developed countries [7, 8]. Asthma occurs as result of hyper and chronic inflammatory immune response to a wide range of antigens in the respiratory tract and is associated with reversible airflow limitations. Numerous reports suggest that the development of asthma is related to the innate immune response, including NK cells, as well as adaptive immune responses [9]. NK cells in the lungs are considered to be regulators of Th1 or Th2 cytokine production, and there is frequently exacerbation of pulmonary eosinophilia during the early induction stage [10].

The effects of asthma on immune responses against virus infection have been discussed in rhinovirus infection, which leads to increased lower airway inflammation [11]. It has been demonstrated that patients with asthma have an increased susceptibility to rhinovirus infection, with more severe lower respiratory tract symptoms and reductions in lung function than healthy subjects who have similar infections [12]. Moreover, the immunological effects have clearly demonstrated that primary bronchial epithelial cells derived from asthmatic patient increase the susceptibility to rhinovirus infection, including increased viral replication and cell lysis, and this is associated with impaired innate immune responses involved in type I IFN production and type III IFN- $\lambda$ production $[13,14]$.

However, the link between asthma and influenza virus infection remains unclear. Therefore, we used an ovalbumin (OVA)-induced asthmatic model mouse to evaluate the susceptibility of the asthmatic and control mice to influenza virus infection and examined their immunological responses against the virus infection.

\section{Materials and Methods}

Mice

Female C57BL/6 mice (6 weeks of age) were purchased from Sankyo Labo Service Co., Inc. (Tokyo, Japan). All mice were housed under specific pathogen-free conditions according to the animal protocol guidelines of the Committee on Animal Care of Tokyo Medical University (protocol no. S-23023).

\section{Influenza Virus and Treatment Protocols}

The influenza virus A/Puerto Rico/8/34 (H1N1) strain was kindly provided by Dr. Kawaoka of the University of Tokyo. Mice were intranasally infected with $20 \mu \mathrm{l}$ of saline containing the virus under anesthesia. Day 0 was defined as the day of viral infection throughout the experiments. The survival rate and body weight were monitored daily until 20 days after the viral infection. The body weight of each mouse on day 0 before the infection was considered to be $100 \%$. In some experiments, mice were intravenously (i.v.) injected with $50 \mu \mathrm{l}$ of anti-asialoGM1 serum (Wako Pure Chemical Industries, Ltd., Osaka, Japan) to deplete NK cells or an equivalent amount of normal rabbit serum (Invitrogen, CA, USA) as a control on days -4 and 0 before the infection.

Induction of Asthma and Measurement of OVA-Specific IgE Production and the Histological Examination of the Trachea and Lungs

Mice were intraperitoneally sensitized with $0.2 \mathrm{ml}$ of phosphate-buffered saline (PBS) including $10 \mathrm{mg}$ of ovalbumin (grade V, Sigma, MO, USA) or PBS as a control every other day for 2 weeks, for a total of seven injections, and were then allowed to rest for 3 weeks. Mice were then intranasally challenged with $20 \mu$ l of PBS containing $0.2 \mathrm{mg}$ of OVA or PBS as a control under anesthesia on days $-3,-2$, and -1 . To estimate the OVAspecific $\operatorname{IgE}$ in the serum and the histology of the respiratory tract and bronchi on day 0 before the virus infection, blood samples, trachea tissues, and lungs were removed from these mice. OVA-specific IgE in the serum was measured using a mouse OVA-IgE ELISA kit (MD Bioproducts, MN, USA) according to the manufacturer's protocol. Trachea tissues and lungs were fixed with $10 \%$ buffered formalin solution for $72 \mathrm{~h}$, and specimens embedded in paraffin wax were cut by a microtome. The sections were stained with hematoxylin-eosin (HE). The slides were analyzed by a histologist without knowledge of the identity of the samples.

Analysis of the Changes in Cell Composition in the Bronchoalveolar Lavage Fluid of Asthmatic Mice

To evaluate the number and types of cells in the bronchoalveolar lavage fluid (BALF) of asthmatic mice, the cells in the BALF were obtained from asthmatic or control mice on day 0 before the infection. Their number and the types of cells were analyzed using an XT-2000iV automatic blood cell analysis instrument (Sysmex Corporation, Hyogo, Japan).

\section{Measurement of the Virus Titer}

The virus titers in the BALF were estimated by modified tissue culture infection dose $50\left(\mathrm{TCID}_{50}\right)$ using hemagglutination as an endpoint. Briefly, confluent Madin-Darby canine kidney cells in 96-well flat bottom plates were washed three times with serum-free Dulbecco's modified Eagle's medium (DMEM), and subsequently, DMEM supplemented with $0.5 \%$ bovine serum albumin (BSA), 
$100 \mu \mathrm{g} \mathrm{ml}^{-1}$ DEAE dextran, $1 \mu \mathrm{g} \mathrm{ml} l^{-1}$ TPCK-treated trypsin, $100 \mathrm{U} \mathrm{ml}^{-1}$ penicillin, and $100 \mu \mathrm{g} \mathrm{ml}^{-1}$ streptomycin (Sigma) was added in each well followed by incubation with serially diluted BALF samples at $33^{\circ} \mathrm{C}$ for $1 \mathrm{~h}$. After gentle washing, the cells were incubated in $200 \mu \mathrm{l}$ of supplemented DMEM in $5 \% \mathrm{CO}_{2}$ at $33^{\circ} \mathrm{C}$ for 3 days. A $150-\mu$ l aliquot of the culture supernatants was then removed and mixed with $0.5 \%$ chicken red blood cell suspension in 96-well round bottom plates at $4^{\circ} \mathrm{C}$ for $3 \mathrm{~h}$. The agglutinated wells were defined as positive wells. The $\mathrm{TCID}_{50}$ for the virus titer was calculated by using the Reed and Muench method [15].

\section{Cytokine Assay of the BALF}

The cytokine concentrations in the BALF supernatants were determined using a mouse Interferon Alpha ELISA Kit (PBL Biomedical Laboratories, NJ, USA) and a mouse IL-12p40 ELISA development kit, mouse IFN- $\gamma$ ELISA development kit, mouse IL-6 ELISA development kit, mouse TNF- $\alpha$ ELISA development kit, mouse IL-4 ELISA development kit, mouse IL-13 ELISA development kit, or a mouse IL-22 ELISA development kit (R\&D Systems, MN, USA) according to the manufacturer's instructions, as described previously [16].

\section{Flow Cytometric Analysis}

The antibodies used in the flow cytometric (FCM) analysis were an anti-CD16/CD32 $\mathrm{Ab}$ (clone: $2.4 \mathrm{G} 2$ ) as an FC blocker, FITC-conjugated anti-CD3 $\varepsilon$ Ab (clone: 145-2 C11), PE-conjugated anti-NK1.1 Ab (clone: PK136), and allophycocyanin-conjugated anti-CD8a Ab (clone: 53-6.7) (BD Pharmingen, CA, USA). In some experiments, PEconjugated $\mathrm{H}-2 \mathrm{D}^{\mathrm{b}}$ Influenza NP tetramer-ASNENMETM (MBL Co., LTD., Aichi, Japan) was used to detect antigenspecific cytotoxic $\mathrm{T}$ cells. The cells in the BALF or spleen were stained in PBS with $1 \%$ BSA and then fixed with $1 \%$ paraformaldehyde, then the FCM analysis was performed on a FACSCalibur flow cytometer with the CellQuest Pro software program.

\section{NK Cell Cytotoxicity Assay}

The NK cell cytotoxicity assays were performed using a coculture system with YAC-1 cells, which are the target cells for mouse NK cells. To prepare effector cells, spleen cells were obtained from asthmatic or control mice on day 0 before the virus infection. After the red cells were lysed, serially titrated effector cells were co-cultured with $1 \times 10^{5}$ cells $\mathrm{ml}^{-1}$ of YAC-1 in DMEM supplemented with $10 \%$ FCS, $100 \mathrm{Uml}^{-1}$ penicillin, and $100 \mu \mathrm{g} \mathrm{ml}^{-1}$ streptomycin in 96 -well round bottom plates at $37^{\circ} \mathrm{C}$ for $6 \mathrm{~h}$. The level of lactate dehydrogenase in the culture supernatants, which is an enzyme released upon cell lysis, was then measured using the CytoTox 96 non-radioactive cytotoxicity assay kit (Promega, WI, USA), and the NK cell cytotoxicity was calculated according to the manufacturer's procedure.

\section{Cytokine Production in Spleen Cells}

Single cell suspensions were prepared from the spleens of asthmatic or control mice on day 6 after the infection. These cells were plated at $2.5 \times 10^{5}$ cells/well in 96-well round bottom plates and stimulated with $1 \mu \mathrm{g} / \mathrm{ml}$ of antiCD3 Ab (clone: 145-2 C11) (BD Pharmingen) in DMEM containing $10 \%$ fetal bovine serum, $100 \mathrm{U} / \mathrm{ml}$ penicillin, and $100 \mu \mathrm{g} / \mathrm{ml}$ streptomycin. The supernatants were collected at $24 \mathrm{~h}$ after starting culture and amounts of IFN- $\gamma$ in the culture supernatants were measured by ELISA kit (R\&D Systems).

\section{In Vivo Killing Assay}

An in vivo killing assay for antigen-specific $\mathrm{CD}^{+} \mathrm{T}$ cells was performed as described [16, 17]. Briefly, single splenocytes from naive mice were stained with a PKH26 red fluorescent cell linker kit for general cell membrane labeling (Sigma). The cells were separated into two equal populations prior to labeling with $50 \mathrm{nM}$ CFSE (Sigma) (defined as CFSE ${ }^{\text {low }}$ ) or $500 \mathrm{nM}$ CFSE (defined as $\left.\mathrm{CFSE}^{\text {high }}\right)$. The $\mathrm{CFSE}^{\text {low }}$ cells were pulsed with $10 \mu \mathrm{M}$ of the nucleoprotein $(\mathrm{NP})_{366-374}$ of the influenza virus PR8 strain, which includes the ASNENMETM peptide sequence-restricted MHC class I $\left(H-2 D^{b}\right)$ [18], for 30 min, while $\mathrm{CFSE}^{\text {high }}$ cells were not pulsed. After washing, the two populations were mixed in equal proportions, and $1 \times 10^{7}$ cells were injected i.v. into the non-infected control mice or mice infected with influenza virus on day 6 . At $4 \mathrm{~h}$ after the injection, the spleen was removed from the mice, and single cells were analyzed by FCM to measure the levels of cytotoxicity. The percent of the killing activity was calculated by the following formula: Percent killing activity $=100-\left\{\left[\left(\% \mathrm{CFSE}^{\text {low }}\right.\right.\right.$ in infected mice / \% CFSE ${ }^{\text {high }}$ in infected mice $) /\left(\% \mathrm{CFSE}^{\text {low }}\right.$ in uninfected mice $/ \% \mathrm{CFSE}^{\text {high }}$ in uninfected mice $\left.\left.)\right] \times 100\right\}$.

Statistical Analysis

The statistical analyses were performed using the Statview ver. 5.0 software program (SAS Institute Inc., NC, USA). The statistical significance of the findings was calculated using the Mantel-Cox log rank test for survival experiments. The unpaired $t$ test was used for the in vitro assays. $p$ values of less than 0.05 were considered to be statistically significant. All values are presented as the means \pm standard deviation. 


\section{Results}

Asthmatic Mice Have Increased OVA-Specific IgE Production, Histopathological Changes, and a Large Number of Infiltrated Cells in the BALF

After OVA sensitization, followed by intranasal OVA challenge in mice, OVA-specific IgE in the serum, pathological appearances in the trachea and lung, and the infiltration of cells in the BALF were observed on day 0 before the virus infection. Neither death of the mice nor acute toxicity, such as a wasted appearance or ruffled fur, was observed during OVA sensitization and OVA challenge. OVA-specific IgE in the serum was significantly produced in mice that were sensitized and challenged with OVA, while no OVA-specific IgE was found in the serum of control mice (Fig. 1a). On histological observation, the basement membrane of the trachea specimens of mice sensitized and challenged with OVA became thickened, and tracheal epithelial cell hyperplasia was observed in mice administered with OVA compared to the control mice (Fig. 1b, upper panels). In addition, as shown in the bottom panels of Fig. 1b, the infiltration of various types of inflammatory cells, especially lymphocytes, was observed in the peribronchial and perivascular areas in the lungs of mice sensitized and challenged with OVA, but not in the control mice. Moreover, excess mucus secreted by bronchial epithelial cells, which is an important pathophysiological indicator of allergic asthma, was also observed in mice sensitized and challenged with OVA. In the analyses of the number or type of cells present in the BALF using the XT-2000iV, the numbers of white blood cells (WBC), neutrophils, monocytes, lymphocytes, eosinophils, or basophils in the BALF of asthmatic mice were higher than those of the control mice after the induction of asthma before the infection (Table I). These results suggest that the mice sensitized to and challenged with OVA would be characterized as having pathological asthma. These mice were, therefore, used as a mouse model of asthma in the present study.

Asthmatic Mice Exhibit Lower Susceptibility to Influenza Virus Infection than the Control Mice

Mice with asthma or control mice were infected with 10 , 100 , or $1,000 \mathrm{pfu}$ of influenza virus, followed by monitoring of the survival rate and body weight daily until day 20. When mice were infected with $10 \mathrm{pfu}$ of the influenza virus, no deaths were observed in either the asthmatic or control mice (Fig. 2a). However, the body weight loss of the asthmatic mice recovered significantly more compared to that of control mice from day 5 to 13 (Fig. 2b). In addition, when mice were infected with 100 pfu of influenza virus, which is a sub-lethal viral dose in control mice, the infected control mice started to die around day 7 , and the final survival rate was $50 \%$ by the end of the observation period. On the other hand, the survival rate of
A

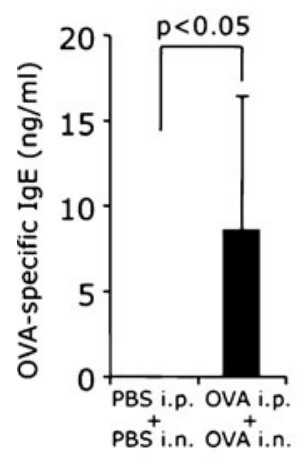

B
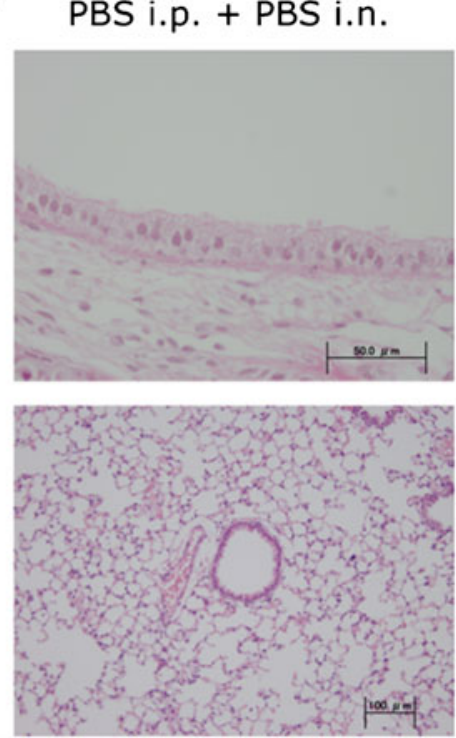

OVA i.p. + OVA i.n.
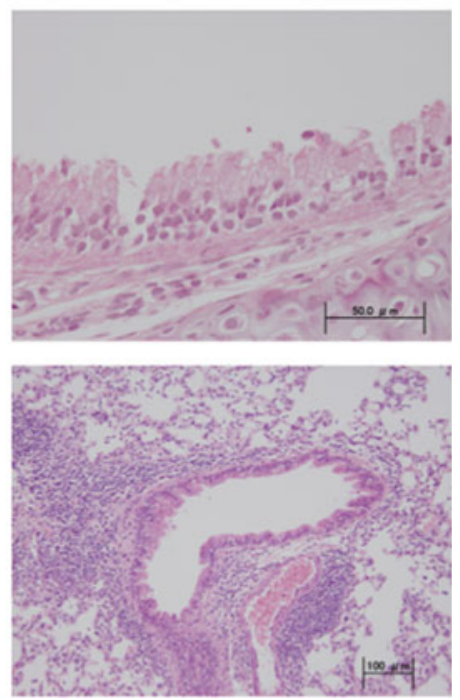

Fig. 1 Asthmatic model mice exhibit increased OVA-specific IgE in their serum and histopathological changes characterized by allergic asthma. C57BL/6 mice were intraperitoneally sensitized with OVA or PBS as a control every other day for 2 weeks, then allowed to rest for 3 weeks. The OVA-sensitized mice or control mice were then intranasally challenged with OVA or PBS on days $-3,-2$ and -1 , respectively. Bloods, trachea tissues, and lungs were collected from the mice on day 0. a The OVA-specific $\operatorname{IgE}$ in the serum was measured by an ELISA assay. b The trachea tissues (upper panels) and lungs (bottom panels) were stained with HE. Six mice were used in each group and similar observations were obtained from each mouse 
Table I Cellular composition in BALF of asthmatic mice before the infection $\left(\times 10^{5}\right.$ cells $)$

\begin{tabular}{|c|c|c|c|c|c|c|}
\hline & WBC & Neutrophil & Lymphocyte & Monocyte & Eosinophil & Basophil \\
\hline PBS i.p. + PBS i.n. & $1.6 \pm 0.3$ & $0.4 \pm 0.2$ & $1.0 \pm 0.3$ & $0.1 \pm 0.1$ & $<10^{4}$ & $<10^{4}$ \\
\hline OVA i.p. + OVA i.n. & $5.6 \pm 2.0^{*}$ & $4.5 \pm 0.9^{*}$ & $2.5 \pm 0.9^{*}$ & $1.0 \pm 0.5^{*}$ & $0.2 \pm 0.2$ & $0.4 \pm 0.2$ \\
\hline
\end{tabular}

The mean and standard deviation from six mice/group are shown

$* p<0.01$ compared to the PBS group

the mice with asthma was $100 \%$, and their body weight loss was also significantly restored compared to the control mice from day 3 to 7 (Fig. 2c, d). When the mice, which were OVA-sensitized without an intranasal challenge with OVA, were infected with 100 pfu of the virus, both the survival rate and the body weight loss were comparable to those of the control mice (data not shown). When the mice were infected with $1,000 \mathrm{pfu}$ of the influenza virus, no significant differences in the survival rate and the recovery of body weight loss were observed between asthmatic
Fig. 2 The survival rate and recovery of body weight loss were improved in asthmatic mice during influenza virus infection. C57BL/6 mice were sensitized and challenged with OVA or PBS. Subsequently, the mice were infected with 10 (a, b), $100(\mathbf{c}, \mathbf{d})$, or $1,000 \mathrm{pfu}(\mathbf{e}, \mathbf{f})$ of influenza virus. The survival rate (a, c, e) and body weight (b, d, f) were monitored daily until day 20 . The body weight data of the dead mouse were excluded from the analysis from the day of death onwards. Twelve mice were evaluated for each group. Similar results were obtained from three independent experiments. ${ }^{*} p<0.05$ compared to the body weight of control mice on the same day. These results are representative of three independent experiments
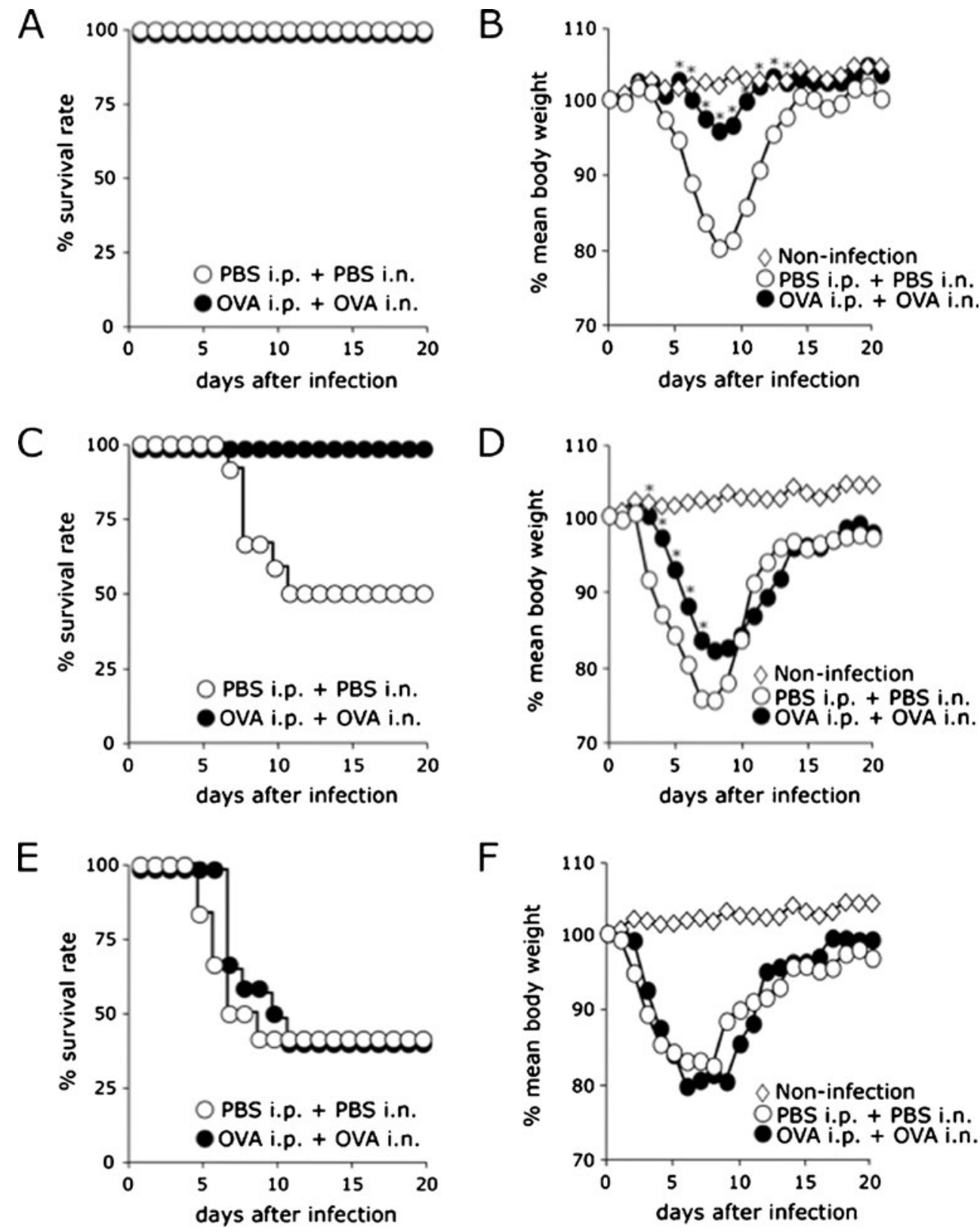

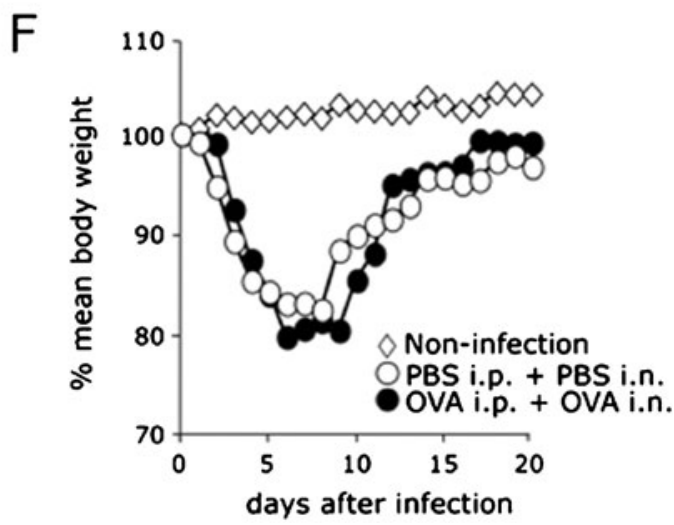


model mice and control mice, thus suggesting that mice with asthma could recover from the pathogenesis of non- or sub-lethal viral infections and that they were more resistant to the effects of the virus than the control mice.

The Virus Titer in the BALF of Asthmatic Mice Is Lower than That of Control Mice

To determine the virus titer in the BALF during infection, mice were infected with $100 \mathrm{pfu}$ of the influenza virus, and the BALF was collected from each mouse on days 2, 4, and 6 after the virus infection. As shown in Fig. 3, the virus titers in the BALF of mice with asthma were significantly almost tenfold lower than those in control mice on days 2 , 4 , and 6 after the virus infection.

Increased Anti-viral Cytokine Production and Decreased Pro-inflammatory Cytokine Production Are Observed in Asthmatic Mice Compared to the Control Mice

Because lower virus titers in the BLAF of asthmatic mice during infection were observed when the mice were administered a sub-lethal virus dose (Fig. 3), the concentrations of cytokines, which can directly and/or indirectly enhance the anti-viral immune responses, such as

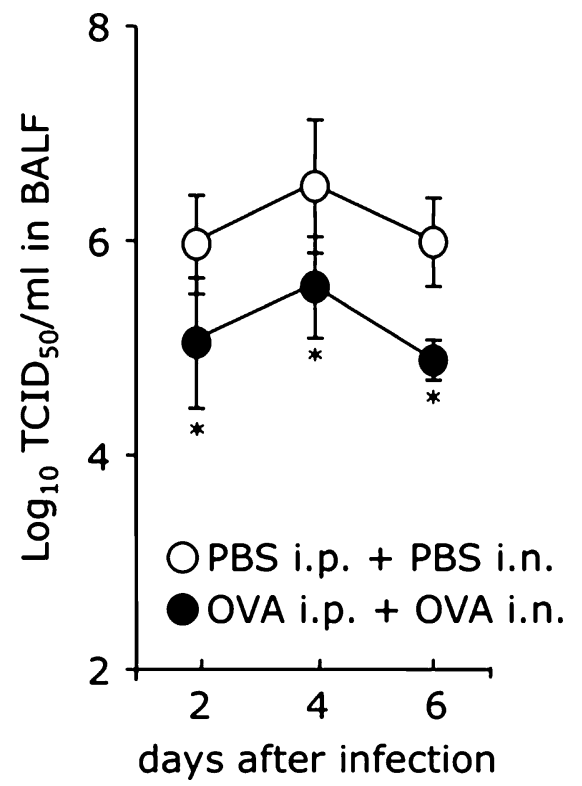

Fig. 3 The virus titer in asthmatic mice is lower than that in control mice after influenza virus infection. C57BL/6 mice were sensitized and challenged with OVA or PBS. The mice were then infected with $100 \mathrm{pfu}$ of the influenza virus. The virus titers in the BALF were calculated by the $\operatorname{TDID}_{50}$ assay on days 2, 4, and 6 after influenza virus infection. Six mice were used in each group. ${ }^{*} p<0.05$ compared to the virus titer of the control mice. Similar results were obtained from two independent experiments
IFN- $\alpha$, IL-12 and IFN- $\gamma$, and pro-inflammatory cytokines, such as IL- 6 and TNF- $\alpha$ in the BALF were determined by ELISA assays. Corresponding to the lower virus titer in the asthmatic mice compared to the control mice, the IL-6 and TNF- $\alpha$ production in the BALF of asthmatic mice was significantly reduced compared to the control mice on day 2 or 6 , respectively (Fig. 4, bottom). In contrast, the concentrations of IFN- $\alpha$, IL-12, or IFN- $\gamma$ in the BALF of asthmatic mice were significantly higher than those in control mice on days 2 and 4 , days 0,2 , and 4 , or day 6 , respectively (Fig. 4, upper panel), thus suggesting that the increased anti-viral cytokines and decreased inflammatory cytokines in asthmatic mice were associated with enhanced viral clearance. On the other hand, the concentration of Th2 cytokines, such as IL-4, IL13, and IL-22, was almost below the limit of detection during the virus infection (data not shown).

NK Cells Migrate from the Spleen to the Lungs of Mice with OVA-Induced Asthma Before Influenza Virus Infection

It is well known that IFN- $\alpha$ and IL-12, which are produced on day 0 before the infection, enhance innate immunity, including NK cell activity, and that IFN- $\gamma$ is produced by activated NK cells. Therefore, in the present study, the frequency and number of NK cells in the BALF were examined. The total numbers of cells significantly increased in the BALF of asthmatic mice compared to those in the control mice (Fig. 5a). Moreover, the frequency and number of NK cells in the BALF of asthmatic mice were also significantly higher than those in control mice, whose number of NK cells in the BALF was below 10,000 cells before the infection (Fig. 5b, c). On the other hand, the frequency and number of NK cells in the spleens of asthmatic mice were significantly lower than those of control mice, although the total number of cells in the spleens of asthmatic mice was comparable to those in control mice (Fig. 5d-f), thus suggesting that the increased number of NK cells in the lungs might have been derived from the spleen via the circulation following the induction of asthma.

The NK Cell Cytotoxicity in Asthmatic Mice Is Enhanced, and the Depletion of NK Cells Abrogates the Resistance of Mice to Influenza Virus Infection

To determine whether NK cells are responsible for the improved survival rate and recovery of body weight against the virus infection, the NK cell cytotoxicity and effects of NK cell depletion were elucidated. As shown in Fig. 6a, the NK cell cytotoxicity in the spleens of asthmatic mice was significantly enhanced with an effector/target ratio of 100:1 compared to the control mice on day 0 before the virus 
IFN- $\alpha$

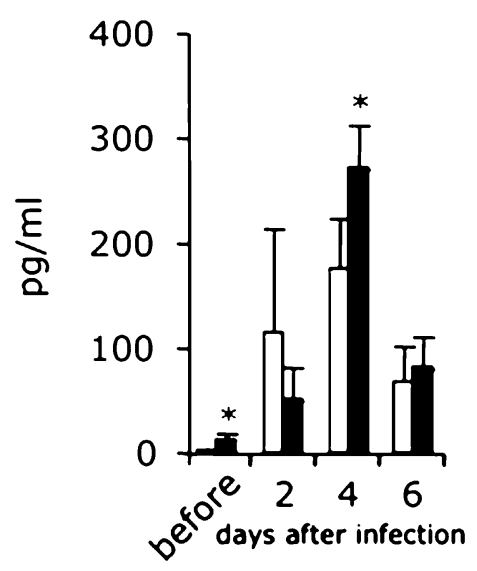

IL-6

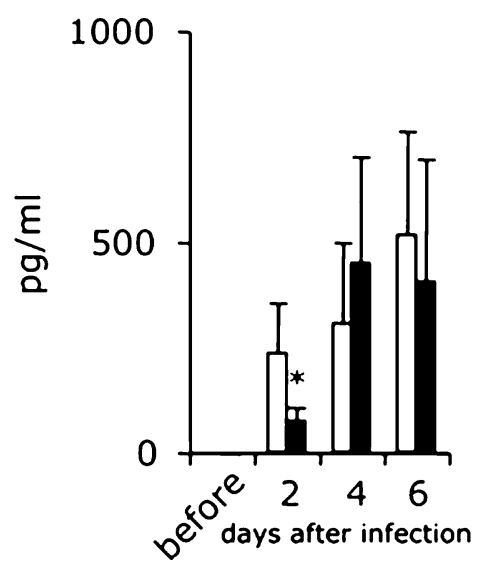

IL-12p40

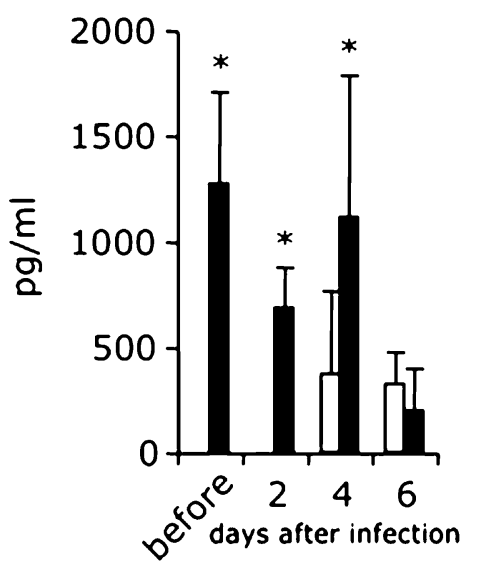

TNF- $\alpha$

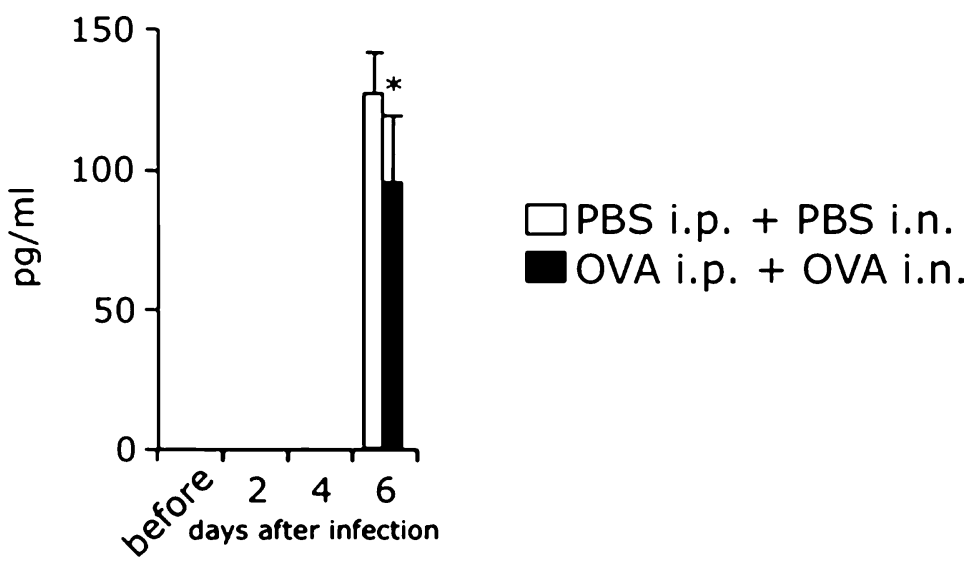

Fig. 4 Differences were observed in the cytokine production between asthmatic mice and control mice during influenza virus infection. C57BL/6 mice were sensitized and challenged with OVA or PBS. Subsequently, the mice were infected with 100 pfu of the influenza virus. BALF samples from the mice were collected on day 0 before viral infection and on days 2, 4, and 6 after influenza virus infection, and the cytokine concentrations in the BALF supernatants were measured by ELISA assays. Six mice were used in each group. These results are representative of two independent experiments. ${ }^{*} p<0.05$ compared to the cytokine production of control mice. These results are representative of two independent experiments

play an important role in the protection against and recovery from influenza virus infection.

The Enhanced IFN- $\gamma$ Production in Asthmatic Mice Reinforced the $\mathrm{CD} 8^{+} \mathrm{T}$ Cell Activity

Because IFN- $\gamma$ production on day 6 after the infection in the asthmatic model mice was significantly increased compared to that in control mice and because spleen cells stimulated with the anti-CD3 $\mathrm{Ab}$ from infected asthmatic mice significantly increased the IFN- $\gamma$ production compared to that of infected non-asthmatic mice (infected asthmatic mice $799 \pm 225 \mathrm{pg} / \mathrm{ml}$ vs. infected control mice $231 \pm 109 \mathrm{pg} / \mathrm{ml}, p<0.01$ ), we examined whether the antigen-specific $\mathrm{CD} 8^{+} \mathrm{T}$ cell killing activity was increased in the asthmatic mice infected with the influenza virus. The 
A

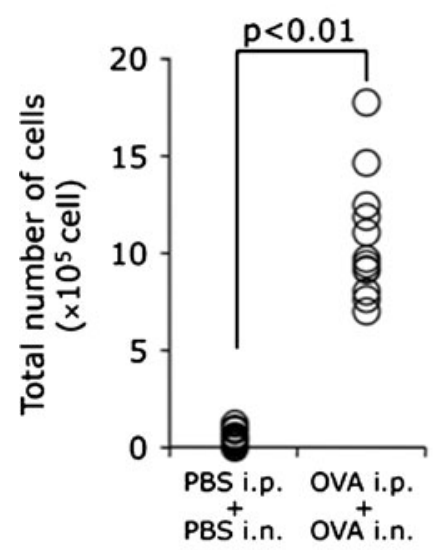

B

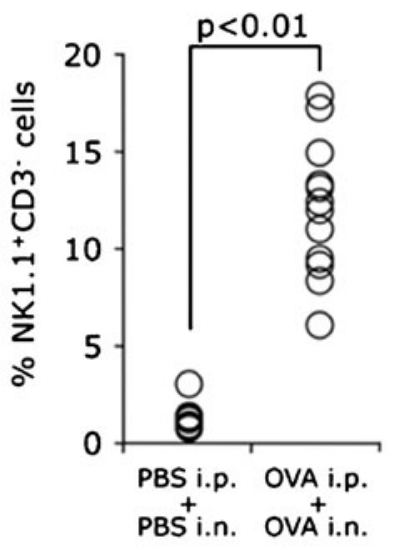

C

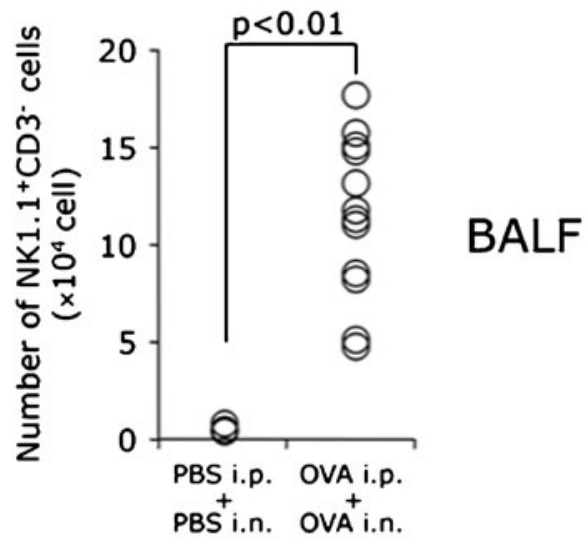

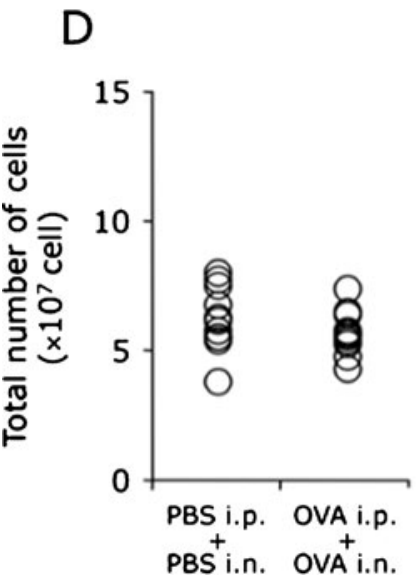

E

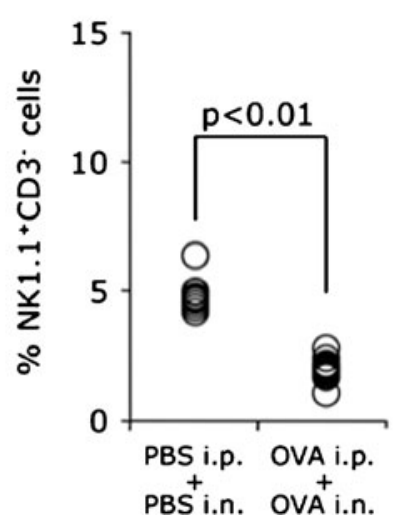

$\mathrm{F}$

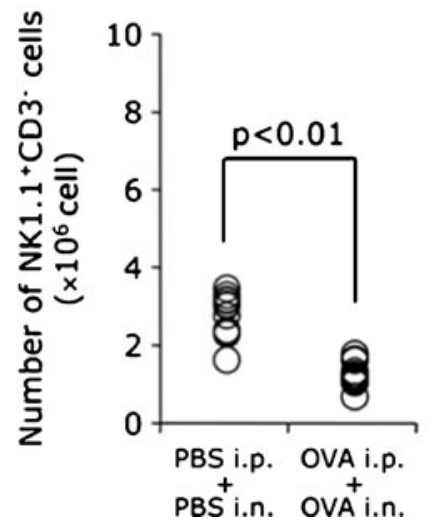

\section{Spleen}

Fig. 5 There are changes in the frequency and number of NK cells in the lungs and spleen following the induction of asthma. C57BL/6 mice were sensitized and challenged with OVA or PBS. The BALF samples or spleens from the mice were collected on day 0 before influenza virus infection. The total numbers of cells in the BALF (a) or spleen (d) were counted under a microscope with $0.25 \%$ trypan blue staining. The frequencies of NK cells in the BALF (b) or spleen (e) were analyzed by FCM. The NK1.1-positive and CD3-negative lymphocytes were identified to be NK cells. The numbers of NK cells in the BALF (c) or spleen (f) were calculated by total cell counting. Twelve mice were evaluated in each group. Similar results were obtained from two independent experiments
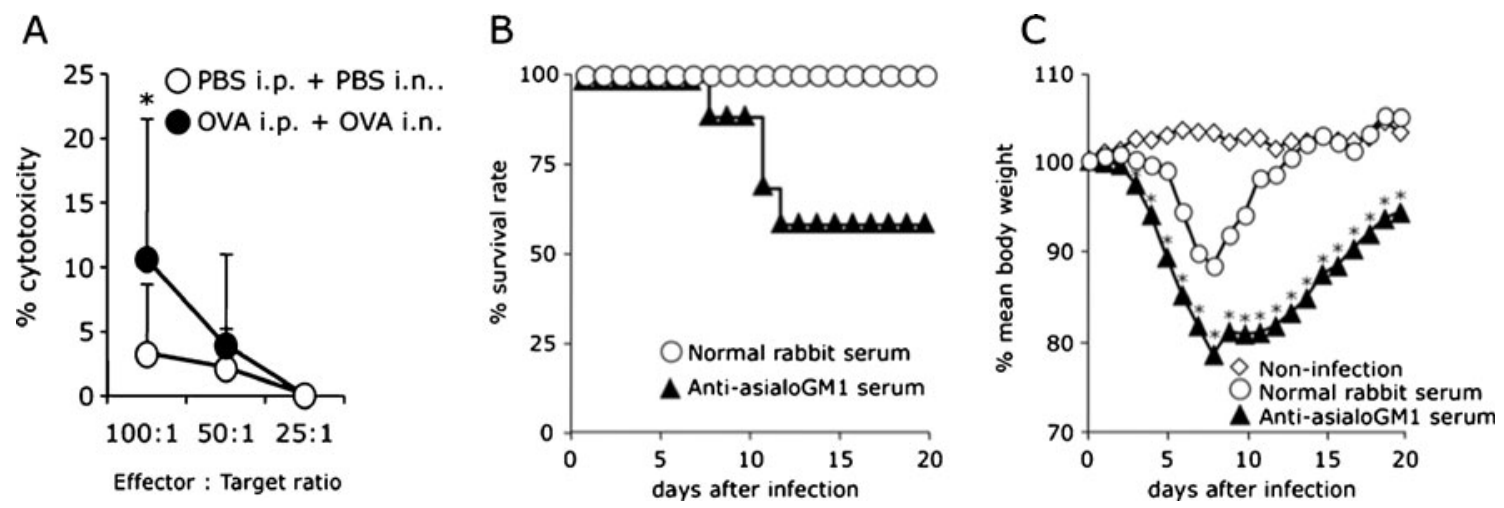

Fig. 6 NK cell cytotoxicity and the effects of NK cell depletion during influenza virus infection. C57BL/6 mice were sensitized and challenged with OVA or PBS. The cells in the spleen were considered to be effector cells and were prepared from asthmatic mice or control mice on day 0 before the infection. The titrated effector cells and $1 \times$ $10^{5}$ cells $\mathrm{ml}^{-1}$ of YAC-1 cells were co-cultured at various effector/ target ratios, as indicated in the figure. The cytotoxicity percentages were calculated as described in the "Materials and Methods" (a).
Asthmatic mice were injected i.v. with normal rabbit serum or antiasialoGM1 serum on days -4 and 0 before the infection. The survival rate (b) and body weight (c) were monitored daily until day 20 . The body weight data of the mice that died were excluded from the day of death onwards. Twelve mice were used in each group. Similar results were observed from two independent experiments. ${ }^{*} p<0.05$ compared to the body weight of control mice on the same day. These results are representative of two or three independent experiments 
frequency and number of antigen-specific $\mathrm{CD} 8^{+} \mathrm{T}$ cells in the spleen were not significantly different between the control and asthmatic mice (Fig. 7a, b). We next examined the killing activity of antigen-specific $\mathrm{CD}^{+} \mathrm{T}$ cells via an in vivo killing assay. As shown in Fig. 7c, the ratio between the peptide-pulsed $\mathrm{CFSE}^{\text {low }}$ cells and unpulsed CFSE ${ }^{\text {high }}$ cells in the spleens of non-infected asthmatic or control mice was equal at $4 \mathrm{~h}$ after the injection with an equal mixture of peptide-pulsed and unpulsed cells. On the other hand, the percentage of peptide-pulsed CFSE ${ }^{\text {low }}$ cells in the spleen decreased in both infected asthmatic and control mice. When the percentages of killing activity of infected asthmatic mice or infected control mice were calculated as described in the "Materials and Methods" section, the in vivo killing activity of the $\mathrm{T}$ cells in the asthmatic mice infected with the virus was significantly increased in comparison to that in control mice infected with the virus (Fig. 7d).

\section{Discussion}

The influenza virus infects the epithelial cells of the upper and lower respiratory tract by binding to sialic acid on the cells and is accompanied by various symptoms in the host. In addition to the virulence of the influenza virus itself, accumulating retrospective studies suggest that asthma is a risk factor associated with the severity of the pathogenesis of influenza virus infection, including infection with the 2009 pandemic influenza A (H1N1) virus [4-6]. On the other hand, it has been reported that asthma is negatively associated with death in patients with confirmed infection with the 2009 pandemic influenza A virus [6]. Therefore, we examined whether asthma affects the pathogenesis of influenza virus infection using asthmatic model mice in vivo. To our knowledge, our findings are the first to demonstrate that asthmatic mice are more resistant to nonand sub-lethal doses of influenza virus as a result of a lower
A

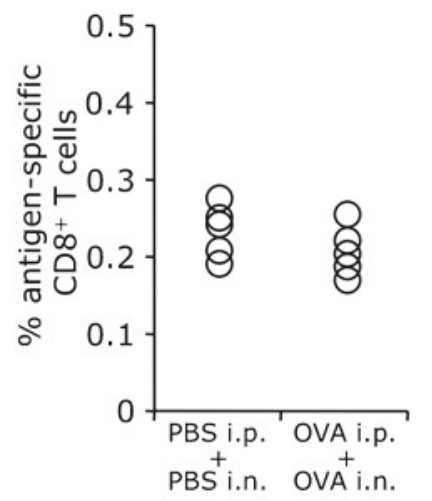

C

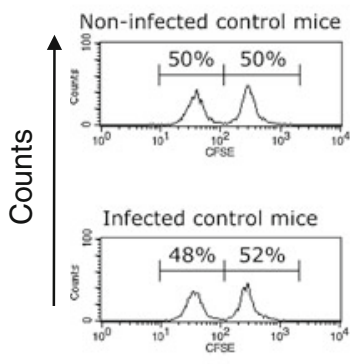

Non-infected asthmatic mice
B

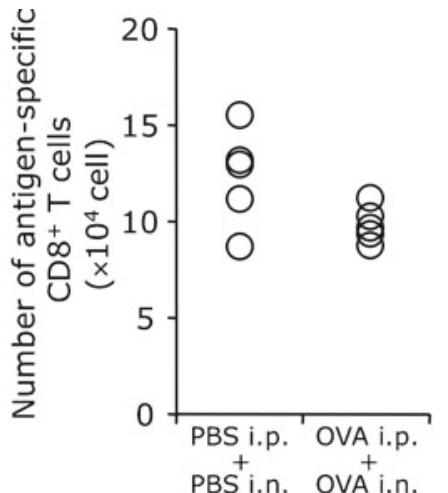

D

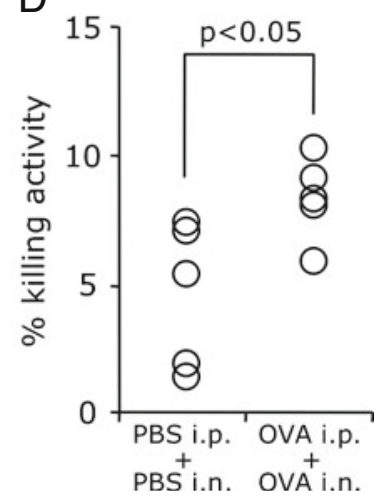

Fig. 7 The antigen-specific cytotoxicity of $\mathrm{CD}^{+} \mathrm{T}$ cells following influenza virus infection. C57BL/6 mice were sensitized and challenged with OVA or PBS. Subsequently, the mice were infected with $100 \mathrm{pfu}$ of influenza virus. An FCM analysis and an in vivo killing activity assay were performed on day 6 after the infection. Cells in the spleen were incubated with $H-2 D^{b}$ Influenza NP tetramer followed by staining with both an anti-CD8 $\mathrm{Ab}$ and an anti-CD3 Ab and analyzed by FCM. Cells that were CD8positive, CD3-positive, and influenza NP tetramer-positive were identified as antigen-specific $\mathrm{CD}^{+} \mathrm{T}$ cells (a). The number of antigen-specific $\mathrm{CD} 8^{+} \mathrm{T}$ cells was calculated by total cell counting (b). Representative FACS data of the in vivo killing assay from noninfected control or asthmatic mice and infected control or asthmatic mice are shown (c). The percentages of in vivo killing activity for asthmatic mice or control mice were calculated as indicated in the "Materials and Methods" (d). Five mice were used in each group. Similar results were obtained from two independent experiments 
viral burden in the lungs than control mice, leading to improvement of their clinical conditions. The immunological mechanisms of the prompt viral clearance in asthmatic mice determined not only increased anti-viral cytokines, such as IFN- $\alpha$ and IL-12, and activated NK cells in the lungs before the virus infection, but also may enhance the antigen-specific $\mathrm{CD}^{+} \mathrm{T}$ cell activity after the infection.

NK cells are normally present in the lungs, and the frequency of NK cells in the lungs is the highest compared to other organs at the steady state [19]. NK cells express a variety of receptors which enable them to exert a primary immunosurveillance function and eliminate cancer cells and infected cells with down-regulated MHC class I [20, 21]. Innate immune responses, including type I IFN and NK cell responses, are essential following influenza virus infection for the direct control of viral replication and to limit the spread of the virus [22]. Moreover, it was recently confirmed that NKp44 and NKp46 on NK cells in humans and natural cytotoxicity receptor 1 on NK cells in the mouse, which is identified as the mouse homolog of human NKp46, recognize influenza virus hemagglutinins expressed on influenza virus-infected cells, leading to rapid recruitment of NK cells and the expansion of the resident NK cells in the lungs [23-27]. In addition, Ly49H or Ly49P, which are expressed on NK cells in mice, recognizes mouse cytomegalovirus (MCMV)-derived proteins m157 and $\mathrm{m} 04$, respectively, and contributes to protecting against MCMV infection [28-31]. These findings suggest that NK cell activation plays crucial roles in the primary defense against the influenza virus or other virus infections [32]. In fact, our current results provide evidence that depletion of NK cells of asthmatic mice before influenza virus infection increases their susceptibility to influenza virus infection, therefore, increasing their morbidity and mortality comparable to that of normal mice. However, it is still unclear whether only NK cells in the lungs of asthmatic mice are fully responsible for the influenza virus elimination, because the depletion of NK cells using anti-asialoGM1 serum depleted not only the local NK cells in the lungs, but also the total body NK cells, including those in the spleen, which is a potent resource of NK cells that migrated to the lungs in this study.

As increased number of NK cells and increased NK cell activity were observed in patients with asthma, however, little is known of their role in the development of asthma $[33,34]$. Korsgren et al. clearly demonstrated the contribution of NK cells in OVA-induced asthma in model mice [10]. When NK cells were depleted by injection of an antibody before sensitization and challenge, their mice had inhibited Th2 responses, such as pulmonary eosinophils, IL-4 and IL-5 production in the BALF, and decreased systemic antigen-specific IgE and IL-4-producing cells, but also had inhibited Th1 responses, including IL-12 production in the BALF and systemic antigen-specific $\operatorname{IgG}_{2 \mathrm{a}}$ and IFN- $\gamma$ producing cells, suggesting that NK cells have the potential to increase in both $\mathrm{Th} 1$ and $\mathrm{Th} 2$ responses during the induction of asthma [10]. Our results also demonstrated that the IFN- $\alpha$ and IL-12 production in the BALF increases in asthmatic following OVA sensitization and challenge before the infection. While IFN- $\alpha$ induces anti-viral protein kinase (PKR), which limits viral replication and induces apoptosis of the infected cells [35], it is well known that IFN- $\alpha$ or IL-12 enhances NK cell cytotoxicity and IFN- $\gamma$ production by NK cells stimulated through NKp46 and NKG2D, respectively [26, 36]. Thus, activated NK cells could promptly eliminate the virus-infected cells and predominantly generate Th1 responses against the virus infection. Although systemic OVA-specific IgE was significantly produced in the asthmatic mice, the Th2 cytokines in the BALF of the asthmatic mice were minimal in our study. This may have been due to the frequency of OVA sensitization and challenge or the injection with/without alum adjuvant used for the induction of asthma. In addition, we measured the cytokines in BALF supernatant, while the supernatants of lung homogenates were used for the cytokine assays in their experiments [10].

IFN- $\gamma$ produced by activated NK cells is important in driving Th1 immune responses, such as antigen-specific $\mathrm{CD}^{+} \mathrm{T}$ cell responses [37]. The ability of virus-specific cytotoxic $\mathrm{CD}^{+}$T cells to thoroughly prevent the influenza virus from escaping from the innate immune response has been demonstrated in $\mathrm{CD}^{+} \mathrm{T}$ cell-deficient mice [38]. Correspondingly, our findings indicated that enhanced IFN- $\gamma$ production in the asthmatic mice would lead to the activation of cytotoxic $\mathrm{CD}^{+} \mathrm{T}$ cells and was associated with preventing the mortality caused by a delay in the clearance of the virus.

Recently, the contamination of commercially available OVA with lipopolysaccharide (LPS) has been reported [39]. Watanabe et al. also indicated that the amount of LPS included in the OVA is sufficient to fully activate endothelial cells, and the mice treated with LPS-free OVA as immunogen without alum adjuvant showed more exacerbation of AHR, lung consolidation, and IgE production than mice treated with commercially available OVA [39]. Moreover, Eisenbarth et al. have reported that inhalation of a low level $(0.1 \mu \mathrm{g})$ of LPS with antigen induces Th2 responses, and a high level $(100 \mu \mathrm{g})$ of inhaled LPS results in Th1 responses to inhaled antigen without alum adjuvant [40]. When commercially available OVA was used to induce asthma in the present study, a high level of IL-12 production was observed in the BALF of asthmatic mice. Therefore, the potential influence of LPS that might have been included in the OVA on the development of asthma could not be ruled out, although the concentration of LPS included in the OVA was not determined. 
In conclusion, our results suggest that asthmatic model mice are more resistant to influenza virus infection as a result of their improved survival rate and more rapid recovery of body weight than control mice. We have also demonstrated one of the mechanisms by which bystander NK cells are activated during the induction of asthma and contribute to protecting the host against influenza virus infection. These activated NK cells may not only to promptly exclude the virus-infected cells by antigen nonspecific bystander effects, but also may increase the antigen-specific $\mathrm{CD}^{+} \mathrm{T}$ cell activity, as demonstrated in the present study. A better understanding of the role of NK cells in asthmatic mice will provide new insights into viral infections.

Acknowledgments We thank Dr. Y. Kawaoka and Dr. T. Horimoto at the University of Tokyo for their generous gift of the influenza virus A/PR/8 strain. This work was supported in part by a Tokyo Medical University Research Grant (to HI).

Conflicts of Interest The authors declare that they have no conflict of interest.

Open Access This article is distributed under the terms of the Creative Commons Attribution Noncommercial License which permits any noncommercial use, distribution, and reproduction in any medium, provided the original author(s) and source are credited.

\section{References}

1. Pribul PK, Harker J, Wang B, Wang H, Tregoning JS, Schwarze J, Openshaw PJ. Alveolar macrophages are a major determinant of early responses to viral lung infection but do not influence subsequent disease development. J Virol. 2008;82:4441-8.

2. Tregoning JS, Yamaguchi Y, Wang B, Mihm D, Harker JA, Bushell ES, Zheng M, Liao G, Peltz G, Openshaw PJ. Genetic susceptibility to the delayed sequelae of neonatal respiratory syncytial virus infection is MHC dependent. J Immunol. 2010;185:5384-91.

3. Tregoning JS, Schwarze J. Respiratory viral infections in infants: causes, clinical symptoms, virology, and immunology. Clin Microbiol Rev. 2010;23:74-98.

4. Gilca R, De Serres G, Boulianne N, Ouhoummane N, Papenburg J, Douville-Fradet M, Fortin E, Dionne M, Boivin G, Skowronski DM. Risk factors for hospitalization and severe outcomes of 2009 pandemic H1N1 influenza in Quebec, Canada. Influenza Other Respi Viruses. 2011;5:247-55.

5. Okada T, Morozumi M, Matsubara K, Komiyama O, Ubukata K, Takahashi T, Iwata S. Characteristic findings of pediatric inpatients with pandemic (H1N1) 2009 virus infection among severe and nonsevere illnesses. J Infect Chemother. 2011;17:238-45.

6. Louie JK, Acosta M, Samuel MC, Schechter R, Vugia DJ, Harriman K, Matyas BT. A novel risk factor for a novel virus: obesity and 2009 pandemic influenza A (H1N1). Clin Infect Dis. 2011;52:301-12.

7. Pelaia G, Vatrella A, Gallelli L, Renda T, Cazzola M, Maselli R, Marsico SA. Respiratory infections and asthma. Respir Med. 2006;100:775-84.

8. Murdoch JR, Lloyd CM. Chronic inflammation and asthma. Mutat Res. 2010;690:24-39.
9. Simpson JL, Brooks C, Douwes J. Innate immunity in asthma. Paediatr Respir Rev. 2008;9:263-70.

10. Korsgren M, Persson CG, Sundler F, Bjerke T, Hansson T, Chambers BJ, Hong S, Van Kaer L, Ljunggren HG, Korsgren O. Natural killer cells determine development of allergen-induced eosinophilic airway inflammation in mice. J Exp Med. 1999;189:553-62.

11. Fraenkel DJ, Bardin PG, Sanderson G, Lampe F, Johnston SL, Holgate ST. Lower airways inflammation during rhinovirus colds in normal and in asthmatic subjects. Am J Respir Crit Care Med. 1995;151:879-86.

12. Corne JM, Marshall C, Smith S, Schreiber J, Sanderson G, Holgate ST, Johnston SL. Frequency, severity, and duration of rhinovirus infections in asthmatic and non-asthmatic individuals: a longitudinal cohort study. Lancet. 2002;359:831-4.

13. Wark PA, Johnston SL, Bucchieri F, Powell R, Puddicombe S, Laza-Stanca V, Holgate ST, Davies DE. Asthmatic bronchial epithelial cells have a deficient innate immune response to infection with rhinovirus. J Exp Med. 2005;201:937-47.

14. Contoli M, Message SD, Laza-Stanca V, Edwards MR, Wark PA, Bartlett NW, Kebadze T, Mallia P, Stanciu LA, Parker HL, Slater L, Lewis-Antes A, Kon OM, Holgate ST, Davies DE, Kotenko SV, Papi A, Johnston SL. Role of deficient type III interferon-lambda production in asthma exacerbations. Nat Med. 2006;12:1023-6.

15. Matumoto M. A note on some points of calculation method of LD50 by Reed and Muench. Jpn J Exp Med. 1949;20:175-9.

16. Ishikawa H, Tanaka K, Kutsukake E, Fukui T, Sasaki H, Hata A, Noda S, Matsumoto T. IFN-gamma production downstream of NKT cell activation in mice infected with influenza virus enhances the cytolytic activities of both NK cells and viral antigen-specific CD8+ T cells. Virology. 2010;407:325-32.

17. Barber DL, Wherry EJ, Ahmed R. Cutting edge: rapid in vivo killing by memory CD8 T cells. J Immunol. 2003;171:27-31.

18. Zhong W, Reche PA, Lai CC, Reinhold B, Reinherz EL. Genomewide characterization of a viral cytotoxic $\mathrm{T}$ lymphocyte epitope repertoire. J Biol Chem. 2003;278:45135-44.

19. Gregoire C, Chasson L, Luci C, Tomasello E, Geissmann F, Vivier E, Walzer T. The trafficking of natural killer cells. Immunol Rev. 2007;220:169-82.

20. Pardoll DM. Immunology. Stress, NK receptors, and immune surveillance. Science. 2001;294:534-6.

21. Welliver TP, Garofalo RP, Hosakote Y, Hintz KH, Avendano L, Sanchez K, Velozo L, Jafri H, Chavez-Bueno S, Ogra PL, McKinney L, Reed JL, Welliver Sr RC. Severe human lower respiratory tract illness caused by respiratory syncytial virus and influenza virus is characterized by the absence of pulmonary cytotoxic lymphocyte responses. J Infect Dis. 2007;195:1126-36.

22. McGill J, Heusel JW, Legge KL. Innate immune control and regulation of influenza virus infections. J Leukoc Biol. 2009;86:803-12.

23. Arnon TI, Lev M, Katz G, Chernobrov Y, Porgador A, Mandelboim O. Recognition of viral hemagglutinins by NKp44 but not by NKp30. Eur J Immunol. 2001;31:2680-9.

24. Mandelboim O, Lieberman N, Lev M, Paul L, Arnon TI, Bushkin Y, Davis DM, Strominger JL, Yewdell JW, Porgador A. Recognition of haemagglutinins on virus-infected cells by NKp46 activates lysis by human NK cells. Nature. 2001;409:1055-60.

25. Mandelboim O, Porgador A. NKp46. Int J Biochem Cell Biol. 2001;33:1147-50.

26. Draghi M, Pashine A, Sanjanwala B, Gendzekhadze K, Cantoni C, Cosman D, Moretta A, Valiante NM, Parham P. NKp46 and NKG2D recognition of infected dendritic cells is necessary for NK cell activation in the human response to influenza infection. $J$ Immunol. 2007;178:2688-98.

27. Gazit R, Gruda R, Elboim M, Arnon TI, Katz G, Achdout H, Hanna J, Qimron U, Landau G, Greenbaum E, Zakay-Rones Z, Porgador A, Mandelboim O. Lethal influenza infection in the 
absence of the natural killer cell receptor gene Ncr1. Nat Immunol. 2006;7:517-23.

28. Smith HR, Heusel JW, Mehta IK, Kim S, Dorner BG, Naidenko OV, Iizuka K, Furukawa H, Beckman DL, Pingel JT, Scalzo AA, Fremont DH, Yokoyama WM. Recognition of a virus-encoded ligand by a natural killer cell activation receptor. Proc Natl Acad Sci U S A. 2002;99:8826-31.

29. Bubic I, Wagner M, Krmpotic A, Saulig T, Kim S, Yokoyama WM, Jonjic S, Koszinowski UH. Gain of virulence caused by loss of a gene in murine cytomegalovirus. J Virol. 2004;78:7536-44.

30. Voigt V, Forbes CA, Tonkin JN, Degli-Esposti MA, Smith HR, Yokoyama WM, Scalzo AA. Murine cytomegalovirus m157 mutation and variation leads to immune evasion of natural killer cells. Proc Natl Acad Sci U S A. 2003;100:13483-8.

31. Kielczewska A, Pyzik M, Sun T, Krmpotic A, Lodoen MB, Munks MW, Babic M, Hill AB, Koszinowski UH, Jonjic S, Lanier LL, Vidal SM. Ly49P recognition of cytomegalovirus-infected cells expressing H2-Dk and CMV-encoded m04 correlates with the NK cell antiviral response. J Exp Med. 2009;206:515-23.

32. Lakshmanan V, Alter G, Altfeld M, Bhardwaj N. Biology of plasmacytoid dendritic cells and natural killer cells in HIV-1 infection. Curr Opin HIV AIDS. 2007;2:189-200.

33. Weissler JC, Nicod LP, Lipscomb MF, Toews GB. Natural killer cell function in human lung is compartmentalized. Am Rev Respir Dis. 1987;135:941-9.
34. Timonen T, Stenius-Aarniala B. Natural killer cell activity in asthma. Clin Exp Immunol. 1985;59:85-90.

35. Balachandran S, Kim CN, Yeh WC, Mak TW, Bhalla K, Barber GN. Activation of the dsRNA-dependent protein kinase, PKR, induces apoptosis through FADD-mediated death signaling. EMBO J. 1998;17:6888-902.

36. Paust S, Senman B, von Andrian UH. Adaptive immune responses mediated by natural killer cells. Immunol Rev. 2010;235:286-96.

37. Robbins SH, Bessou G, Cornillon A, Zucchini N, Rupp B, Ruzsics Z, Sacher T, Tomasello E, Vivier E, Koszinowski UH, Dalod M. Natural killer cells promote early CD8 T cell responses against cytomegalovirus. PLoS Pathog. 2007;3:e123.

38. Bender BS, Croghan T, Zhang L, Small Jr PA. Transgenic mice lacking class I major histocompatibility complex-restricted $\mathrm{T}$ cells have delayed viral clearance and increased mortality after influenza virus challenge. J Exp Med. 1992;175:1143-5.

39. Watanabe J, Miyazaki Y, Zimmerman GA, Albertine KH, McIntyre TM. Endotoxin contamination of ovalbumin suppresses murine immunologic responses and development of airway hyperreactivity. J Biol Chem. 2003;278:42361-8.

40. Eisenbarth SC, Piggott DA, Huleatt JW, Visintin I, Herrick CA, Bottomly K. Lipopolysaccharide-enhanced, toll-like receptor 4dependent T helper cell type 2 responses to inhaled antigen. J Exp Med. 2002;196:1645-51. 